\title{
ROBUSTNESS EVALUATION OF PRODUCT CONCEPTS BASED ON FUNCTION STRUCTURES
}

\author{
Goetz, Stefan; Hartung, Jonas; Schleich, Benjamin; Wartzack, Sandro \\ Friedrich-Alexander-Universität Erlangen-Nürnberg
}

\begin{abstract}
Due to the varying environment conditions as well as the manufacturing induced deviations, the properties of products vary. In order to still meet the increasingly tightening of functional requirements, tolerancing as well as Robust Design practices became integral parts of the product development. However, despite the fact that the robustness of a product is mainly determined by its conceptual design in early design stages, these activities are usually carried out at the end of the design process. In order to overcome this shortcoming, this contribution shows a method that supports the selection of robust principal solutions and thus contributes to the design of product concepts, which are less sensitive to variations. The novelty lies in the adaption and combination of robust design criteria for the quantitative robustness evaluation in the conceptual design stage. First the product characteristics, which are relevant for the product robustness are determined on the basis of the function structure. By using an adopted VMEA and a newly developed evaluation matrix, this allows a thorough robustness evaluation of product concepts. The method is exemplary shown for a lifting table.
\end{abstract}

Keywords: Robust design, Conceptual design, Tolerance representation and management, QFD, VMEA

Contact:

Goetz, Stefan

Friedrich-Alexander-Universität Erlangen-Nürnberg

Engineering Design

Germany

goetz@mfk.fau.de

Cite this article: Goetz, S., Hartung, J., Schleich, B., Wartzack, S. (2019) 'Robustness Evaluation of Product Concepts based on Function Structures', in Proceedings of the 22nd International Conference on Engineering Design (ICED19),

Delft, The Netherlands, 5-8 August 2019. DOI:10.1017/dsi.2019.359 


\section{INTRODUCTION}

The increasing complexity of products in combination with the striving for short development times requires the application of structured product design processes. Accompanied by virtual methods, such systematic approaches aim at a high product quality according to the "right first time" philosophy (Göhler, 2017). In order to implement this philosophy, various design process models splitting the design process into several phases such as planning, conceptual, preliminary and detail design (Pahl et al., 2007) have been proposed. The progressive accumulation and transformation of initially qualitative into quantitative product information steadily reduces the space of possible solutions and thus enables the virtual validation of products in late design stages, labelled as "further design process" in Figure 1. However, most of the product lifecycle costs are already fixed in these stages, so that potential design changes become expensive (Haskins, 2006). Therefore, most disciplines aim at a frontloading of the virtual validation. Although it has been shown that in particular the conceptual design has an enormous influence on the robustness and thus on the performance of the final product (Ebro et al., 2012), it is still common practice to consider variations not before the late stages (Göhler, 2017). The reason for this can be found in the lack of feasible approaches supporting the designer in the selection of robust product concepts (Gremyr and Hasenkamp, 2011). In order to facilitate the complex choice of concepts, the design process models propose a breakdown to the level of principal solutions. This enables a differentiated evaluation and corresponding selection of the individual principal solutions, each representing an implementation of a single function. The combination of these selected solutions finally results in the product concept, as shown in Figure 1.

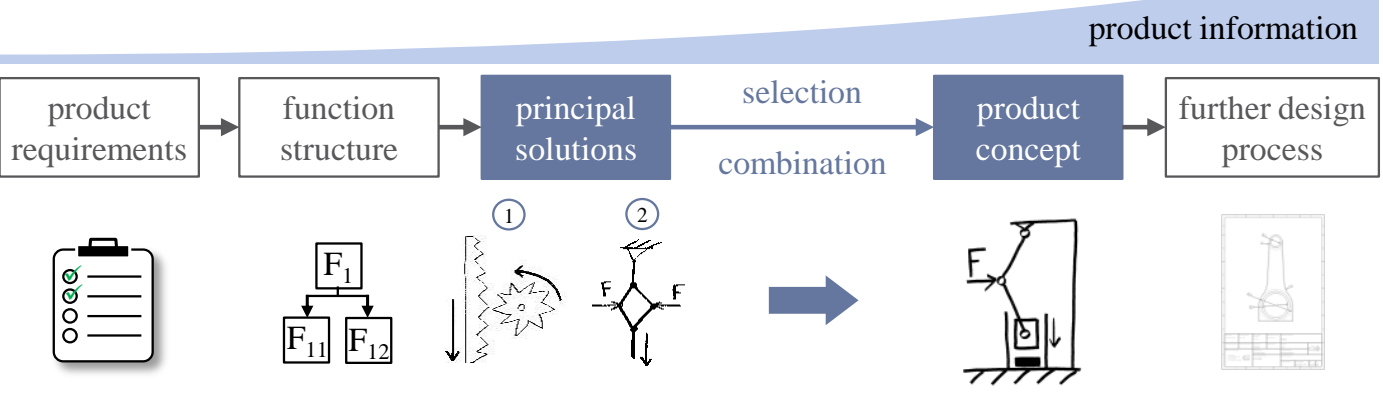

Figure 1. Selection of principal solutions in the product development process

In order to overcome the uncertainty of the evaluation and selection process for principal solutions, numerous promising multi-criteria evaluation approaches (Justel et al., 2007; Zhai et al., 2009; Ayağ, 2016) have been developed beyond standard methods such as the cost-benefit analysis. These approaches enable a reliable evaluation based on qualitative information available in the conceptual design and thus foster the impartial comparison of alternative solutions. Although the existing methods are established in product development, a systematic consideration of possibly occurring variations is missing. With the aim to overcome this, the approach proposed in this contribution enables the robustness evaluation of design concepts or rather principal solutions and thus adds an essential aspect in the multi-criteria evaluation for the concept choice. In this context, the research question has to be clarified to what extent a quantitative robustness evaluation can be carried out on the basis of the limited information available in these early product development phases in order to achieve objectively reliable results.

The paper is structured as follows. First, related work considering Robust Design and quality management in the context of the conceptual design is presented. Subsequently, section 3 describes the basic idea of the proposed robustness evaluation of principal solutions as well as the concrete procedure. After applying the proposed approach on the conceptual design of a lifting table in section 4 , the contribution closes with a conclusion and an outlook.

\section{QUALITY MANAGEMENT AND ROBUST DESIGN FOR CONCEPTS}

Since the impact of variations on the product performance is widely acknowledged, numerous approaches have been evolved in recent years to consider them during the product development process. The corresponding activities can be united under the global concept of quality management with a special discipline of this being Robust Design, which is primarily focusing the design and 
engineering process. While concrete quantitative methods are prevailing in this context, the overall quality management considers further aspects mainly based on qualitative information. Accordingly, as indicated in Figure 2, approaches from the quality management as well as the Robust Design are beneficial for the robustness evaluation in the conceptual design.

Due to its long history, quality management has changed significantly. Starting from the approach of improving quality through inspection, the discipline has evolved to the comprehensive Total Quality Management (Pfeifer, 2002). Thus, this extended approach is not a single method but rather a comprehensive strategy or philosophy. This includes a thorough linkage of the Quality Management with other business (Kim-Soon, 2012) or engineering processes, such as the product development (Hermans and Liu, 2013; Song and Su, 2015). Thereby the Quality Function Deployment (QFD) is of particular importance (Cheng Lim et al., 1999; Akao, 2004). The QFD approach enables the translation of customer requirements into technical requirements for each stage of the product development and production (P. Sullivan, 1986). Since the first phase of the QFD enables the generation of quantifiable design-independent product characteristics based on the qualitative customer requirements (Zare Mehrjerdi, 2010), it is of particular importance in the beginning of the design process. However, since the QFD evaluation process is usually performed in cross-functional design teams (Hoffman, 2014), trade-offs typically arise.

In order to overcome this problem, the Failure Mode and Effect Analysis (FMEA) can be used in the QFD for the evaluation of the risk associated with design decisions (Almannai et al., 2008). The qualitative method incorporates the severity of the effect as well as the probability and detectability of failures and combines these criteria in a common risk priority number (RPN) (McDermott et al., 2009). Thus, it reveals the failure sensitivity of the analyzed system at an early stage in the product development and fosters the development of non-failure-prone products. In this way, the FMEA can be used for the identification of critical technical specifications with regard to occurring variation (Thornton, 2004). Furthermore, the Variation Mode and Effect Analysis (VMEA) adopted the general idea of the FMEA in order to evaluate the susceptibility of the system to variation shifting the method into a Robust Design context (Johansson et al., 2006). In a similar fashion, the Uncertainty Mode and Effect Analysis (UMEA) takes into account uncertainties from the entire product life cycle and thus incorporates a multitude of aspects including epistemic uncertainties (Engelhardt et al., 2009). Although these approaches are designed for the application based on qualitative information, their concrete application within the framework of concept design is often considered as difficult.

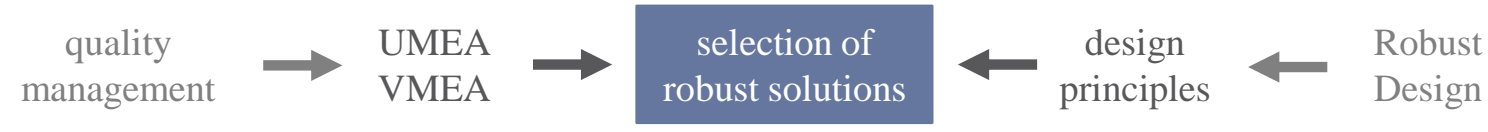

\section{Figure 2. Different views on the consideration of the robustness of principal solutions}

Therefore, the Robust Design methods dealing with the impact of variation from a design perspective have been established. Their general idea is to design a product in such a way that it is least sensitive to variation and thus meets the required performance at the lowest possible production costs (Dehnad, 1989). Taguchi considers the Robust Design as an accompanying process of the product development. Accordingly, the process is separated in the three stages system design, parameter design and tolerance design (Taguchi et al., 1990). The latter two steps focus the optimization of the parameters and tolerances with respect to a low sensitivity in the late design stages, as exemplary shown by (Schleich et al., 2014; Heling et al., 2018). In contrast, the prior system design deals with the product design itself. Although this stage of the Robust Design is often neglected in practice (Gremyr and Hasenkamp, 2011), it has a significant influence on the robustness and is therefore of particular importance in the context of this contribution. Thus, tolerance graphs have already been successfully used for a qualitative robustness evaluation on the basis of principle sketches (Goetz et al., 2018). However, due to the lack of quantitative information in the early stages of the product development, it is difficult to quantify the robustness of a design prior to the final geometric definition (Taguchi et al., 2005). Therefore, experience-based design principles have been established in order to lead to a Robust Design when consistently applied in the product development. As the overview in Figure 3 indicates, the basic idea of the numerous principles is to make the design safe and at the same time as simple as possible.

Since the concept already defines a majority of the product design, these principles, which primarily concentrate on the late design stages, can also be applied during the definition of the product concept 
(Ebro and Howard, 2016). Although the principles are proper criteria for the rough estimation of the robustness of principal solutions, it is difficult to unite and quantify them. In this context, as an extension to the consideration of the coupling of systems, the Contradiction Index was developed (Göhler and Howard, 2015). Beyond the simple consideration of couplings, the approach takes into account the contradictoriness of the functional requirements and the technical specifications (Göhler and Howard, 2015). In combination with a scoring scheme, the approach provides a quantitative statement of the contradiction and thus allows an easy identification of the concept with least contradictoriness. Furthermore, an alternative robustness index can be calculated based on the physical effects underlying a principal solution (Mathias et al., 2011). Thus, the formula expression describing the effect enables a quantitative sensitivity statement of the basic concept.

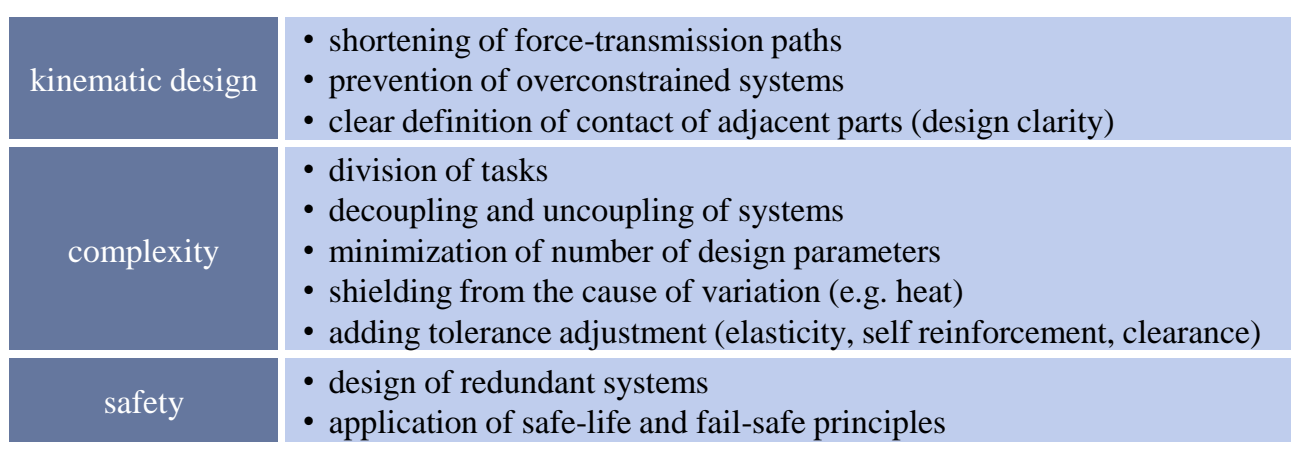

Figure 3. Collection of Robust Design principles and rules (Andersson, 1997; Matthiassen, 1997; Suh, 1998; Söderberg and Lindkvist, 1999; Ebro et al., 2012; Ebro and Howard, 2016)

Although these individual approaches are beneficial, they are limited to single Robust Design principles. Therefore, a comprehensive approach, similar to the FORFLOW process model for interdisciplinary product development (Krehmer et al., 2010), considering the multitude of Robust Design principles is missing. In order to realize the necessary quantification of the Robust Design principles from Figure 3, their coupling with quality management driven methods, which usually work with qualitative information, is useful in the context of a comprehensive robustness evaluation in the conceptual design.

\section{ROBUSTNESS EVALUATION OF PRINCIPAL SOLUTIONS}

In this respect, the gradual approach shown in Figure 4 enables the determination of Robustness Indices (RI) for principal solutions based on the function structure and thus fosters the development of robust product concepts. The procedure starts with the definition of Functional Key Characteristics (FKCs) inspired by the definition of Key Characteristics (Thornton, 2004). A FKC describes a preferably quantifiable product specification whose variation has a major influence on the fulfilment of the functional requirements and is therefore important in the context of the Robust Design evaluation. In contrast to the common design process, the FKCs are already derived from the function structure using the QFD, for example. Although this step enables the limitation to the significant characteristics according to the Pareto principle, a further weighting of the remaining FKCs reflecting their relevance with regard to the product is useful in the context of Robust Design.

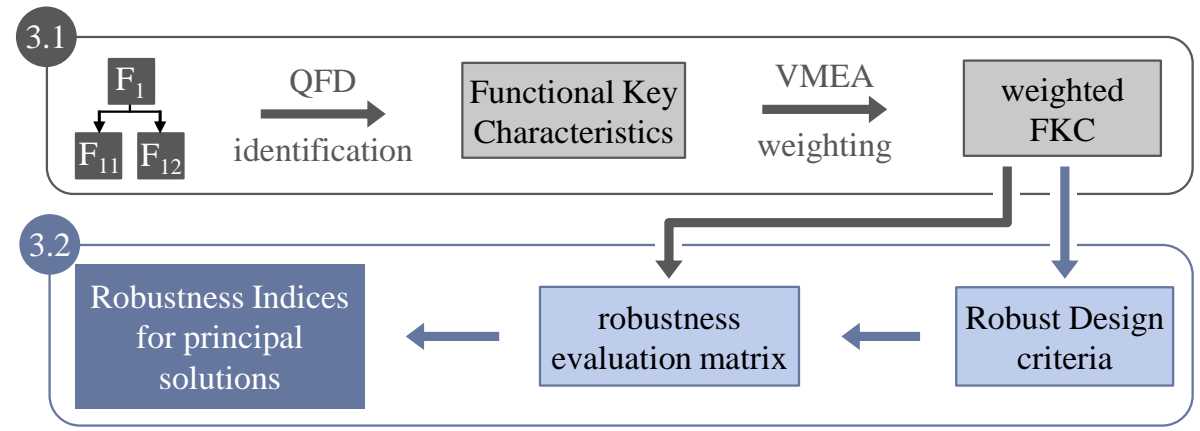

Figure 4. General framework for the robustness evaluation of principal solutions

The weighting resulting from the adapted VMEA (see section 3.1) is the first input factor for the robustness evaluation matrix. The second important input results from the application of the Robust 
Design criteria to the evaluation of principal solutions with regard to the previously defined FKCs. Depending on the complexity of the product and the desired level of detail in the conceptual design stage, the robustness evaluation is also applicable for partial concepts representing a combination of individual principal solutions. The result of the robustness evaluation matrix (see section 3.2) is an individual Robustness Index (RI) for each principal solution respectively partial concept. This quantitative index representing the sensitivity of the principal solutions, can be used as an additional factor in the multi-criteria evaluation process and can thus contribute to a selection of concepts with regard to among others technical, economic and in addition robustness aspects. The individual steps for the evaluation of the RI, are discussed in the following sections, in which the necessary preliminary work and the subsequent robustness evaluation are explained in detail.

\subsection{Definition of functional key characteristics}

In order to realize a smooth integration of the proposed robustness evaluation of principal solutions in the product development, the process starts with the function structure usually available at the end of the planning phase according to Pahl and Beitz. Depending on the level of detail, the given function structure is first broken down to the subfunction level. In the sense of an efficient product development, this step should be limited to the functions relevant for the fulfilment of the customers' requirements. In order to make this distinction, the specific questions of the QFD, which are already established in the early design stages, are beneficial. Since the QFD is a multi-stage process that goes beyond the translation of customer wishes into technical requirements, the method can also be used for the identification of the FKCs, as shown in Figure 5. The thorough linkage with the customer's voice also ensures the limitation to relevant characteristics. Furthermore, the early identification of the FKCs on this basis guarantees that they are defined independently of the concepts subsequently developed in the design process and that the FKCs are thus neutral for the subsequent robustness evaluation.

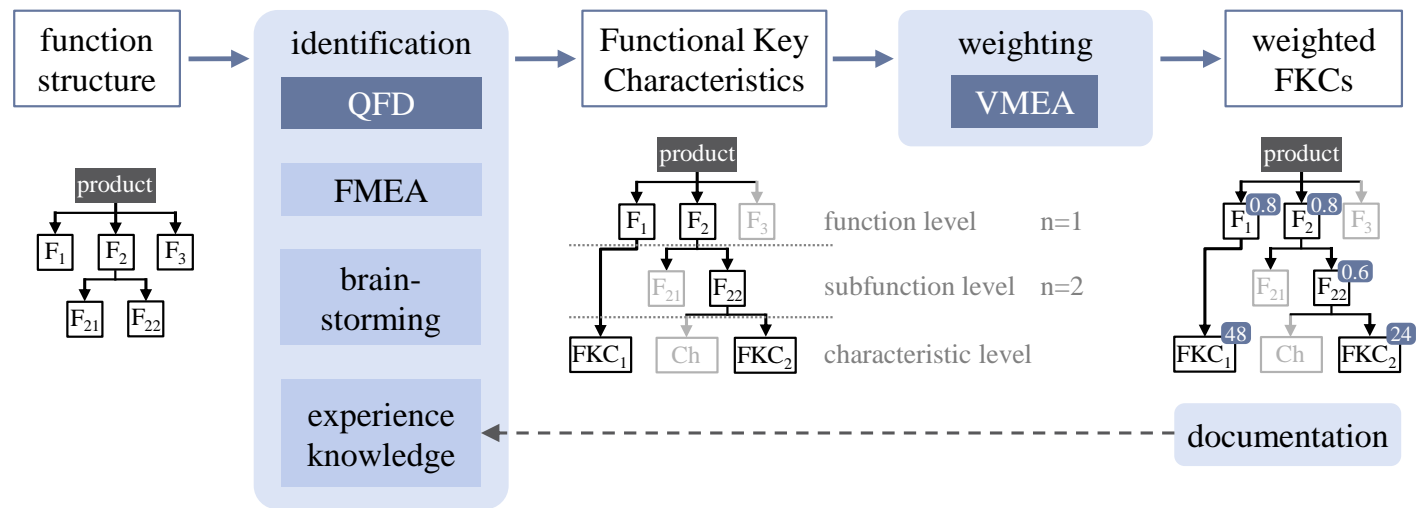

Figure 5. Workflow for the weighting of Functional Key Characteristics (FKCs)

In order to avoid the over-prioritization of individual characteristics, the QFD should be carried out in multidisciplinary teams preferably with experience in the identification of FKCs. Since this process can nevertheless be challenging, established methodological approaches such as brainstorming or FMEA can be used in the QFD framework for the determination of potential characteristics and the assessment of their significance. While this step already preselects the relatively few relevant characteristics, it is inevitable for the subsequent robustness evaluation based on the FKCs to quantify their significance with regard to the product.

Therefore, in order to support the challenging weighting of FKCs, the VMEA was adapted to the abstract character of this early design stage. For this purpose, both the factors and the formula for calculating the Variation Risk Priority Number (VRPN) were customized for good applicability, see Equation 1.

$$
V R P N=\prod_{i=1}^{n} S_{i} \cdot I \cdot P \cdot C
$$

The first additional term represents the significance of the respective function or subfunction of the lowest level of the function structure with regard to the entire product. While $n$ defines the number of existing levels of the function structure (see Figure 5), the factor $\mathbf{S}$ varying from 0 to 1 describes the significance (from low to high) of the respective functions and subfunctions with regard to the higherlevel function or product. This gradual procedure, in which only the reference to the next level in the 
function structure has to be taken into account, allows an easy determination of the significance of individual functions, even in complicated systems. In particular, this is beneficial for assessing the importance of individual FKCs with regard to the overall product. Instead of having to consider superordinate abstract relationships, the designer can focus on evaluating the impact I of the FKC on the corresponding function. Another fundamental factor for the calculation of the VRPN is the probability $P$, which reflects how likely it is that variations of the FKC occur. According to the VMEA proposed by (Johansson et al., 2006) the scale for $I$ and $P$ ranges from 1 to 10 , where 1 corresponds to a low impact respectively probability and 10 corresponds to a high sensitivity. In contrast to the conventional VMEA, the additional factor $C$ allows the consideration of potential corrective adjustments and is thus particularly adapted to the application of the method in the context of the subsequent robustness evaluation. This additional factor is particularly beneficial for experienced users incorporating expertise from predecessor products. For example, it can be taken into account whether an unacceptable variation of the size is already detected during the manufacturing process or only during use by the customer. Although this aspect is important for determining a reliable VRPN, there is a risk of unconsciously focusing on individual concepts and thus distorting the results. Therefore, in order to avoid an overestimated effect and thus an incorrect rating, the scale for $C$ is limited to a range from 0.75 to 1 .

Thus, the resulting VRPN from Equation 1 indicates how likely it is that variations of individual FKCs have a negative effect on the product. The weighted function structure including the significance of relevant functions and subfunctions as well as the results of the modified VMEA for each FKC is the fundamental basis for the robustness evaluation described in the following section. Moreover, a thorough preparation and documentation of the results contributes to the formalization of the usually implicit experience knowledge and thus contributes to a significant acceleration and partial automation of the evaluation process, particularly when analyzing frequently recurring functions. Finally, this also contributes to a better traceability and therefore facilitates potential design changes.

\subsection{Determination of robustness Indices for each solution}

As already indicated at the beginning of section 3, the robustness evaluation matrix, which is shown in Figure 6, is the main element for the determination of the Robustness Indices (RI). Depending on the system under investigation, suitable Robust Design criteria must initially be selected from the overview shown in Figure 3. Thus, for example, the safety design criteria are primarily applicable to safety-relevant products such as aircrafts and can therefore often be neglected in the robustness evaluation. Although this modular approach allows the limitation to relevant criteria, their selection can be challenging for inexperienced users at the beginning. Table 1 therefore offers a proposal that provides a good basis for a first evaluation of most common mechanical engineering products.

Table 1. Suggested Robust Design criteria with weighting and associated aspect of interest

\begin{tabular}{|l|c|r|}
\hline Robust Design criterion & weighting & aspect of interest \\
\hline degree of constraint & 3 & FKC \\
\hline number of parameters & 2 & FKC \\
\hline coupling & 2 & function/product \\
\hline
\end{tabular}

In order to realize the intended combination of the various criteria in a common evaluation, their weighting $(1,2$ or 3$)$ is added in the matrix shown in Figure 6. A higher weighting thereby represents a greater significance of the criterion for the product. Since the choice and weighting of Robust Design criteria strongly depends on the product being analyzed and partly reflects the products requirements, an individual adaption makes sense for a detailed evaluation. Furthermore, as the right column in Table 1 already indicates, the multi-criteria evaluation means that some of the criteria refer not only to individual FKCs but to the entire function or the product. Thus, the criterion "coupling" at product level takes into account the extent to which the functions of the product in the respective solution alternatives examined are coupled with each other, for example by function integration. On the other hand, the evaluation of the coupling on function level considers the interactions of FKCs assigned to respective functions.

Each of the concept solutions evaluated in the matrix (see Figure 6), by default represents a combination of different individual principal solutions. In order to unify their evaluation with regard to the different Robust Design criteria for the corresponding different aspects of interest to a single RI per solution, the VRPN and the values $\mathrm{S}$ from the VMEA described in the previous section are essential. These values enable the evaluation to be carried out at the FKC and function level and by weighting allow a 
subsequent transfer of the results to the product level. Since this means that the key figures (highlighted in cream) calculated for each criterion and solution are on product level, they can easily be summed up to the common RI.

However, in order to calculate the RI, it is first necessary to transfer the usually qualitative robustness evaluation according to the Robust Design criteria to a quantitative evaluation number (highlighted in light grey in Figure 6). For this purpose, the following three valuation methods are provided among others: ranked order, value table and FMEA. In the simplest scenario ranked order, the different solutions are ordered by descending robustness according to the respective criterion, so that the key figure corresponds to the rank. For a finer gradation of the criteria evaluation numbers, a value table can be used for translating the qualitative statements into standardized numerical values. For example, the criterion degree of constraint allows a simplified distinction between overconstrained (10), overconstrained with potential compensation by adjustment or elasticity (5) and not overconstrained (0) and thus an easy definition of the evaluation numbers. Alternatively, a further FMEA can be used for an even more specific assessment. Although the different valuation methods, in the order given above, allow an increasing differentiation between the individual solutions, their application requires an increasing degree of experience and knowledge about the solutions studied. Since the consistent use of a single valuation method within a Robust Design evaluation matrix is essential for reliable results, the ranked order is to be preferred in case of doubt.

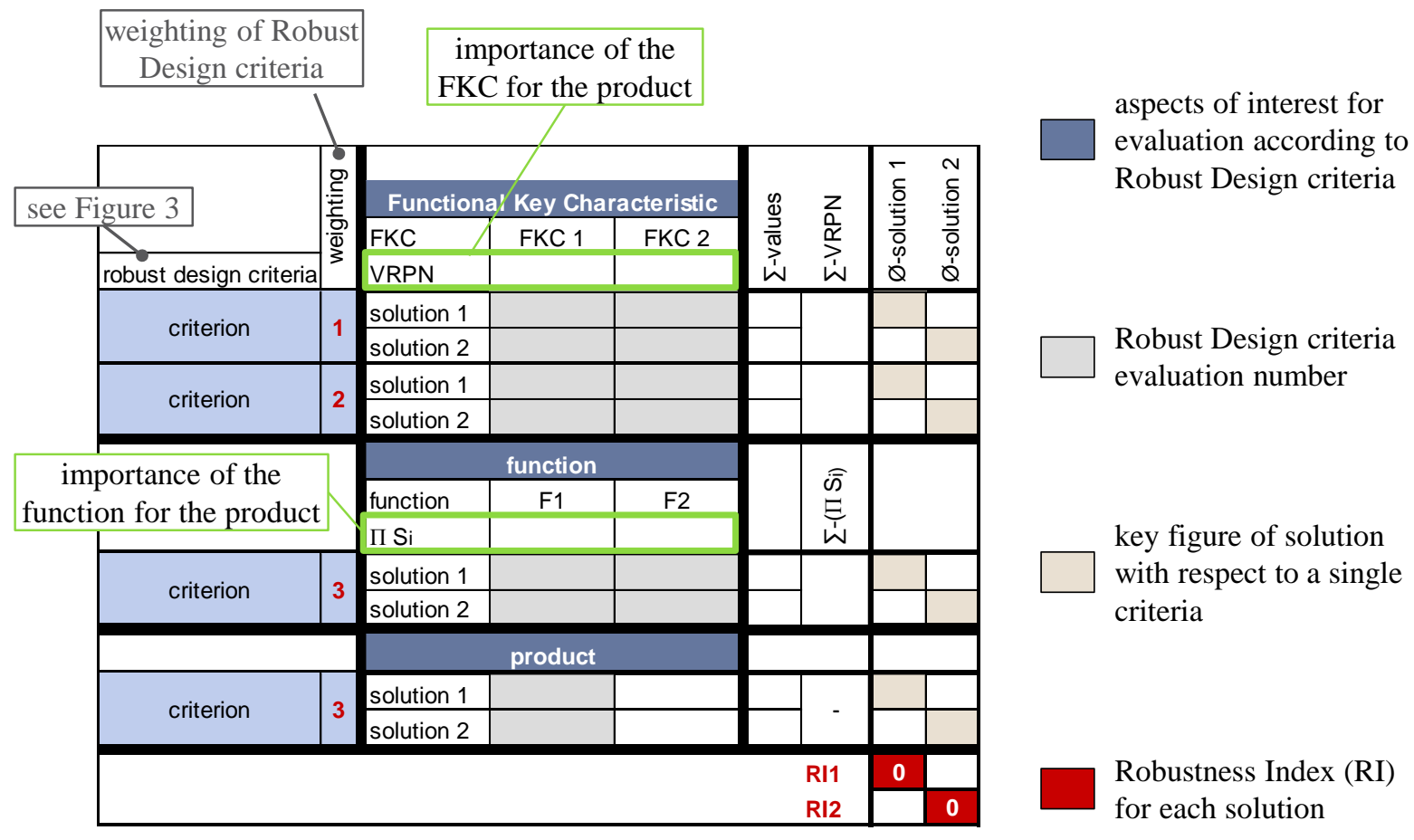

Figure 6. Robust Design evaluation matrix for the determination of the Robustness Index (RI)

This means that quantitative evaluation numbers (highlighted in light grey in Figure 6) are defined for each criterion, solution and aspect of interest, so that the calculation of the RI for the various solutions can be performed automatically. For this purpose, the evaluation numbers are first multiplied by the corresponding weighting and, if available, by the VRPN or S. By then summing these products column by column and dividing them by the respective sum of VRPN and S, the key figures of the individual solutions are obtained with respect to the respective criterion. The RI resulting from its line by line summation reflects the robustness of the investigated solutions. The lower the RI, the less sensitive the solution is to potential deviations.

\section{CASE STUDY}

In order to enhance the traceability of the workflow and to demonstrate the benefits, the proposed approach is exemplary applied to the development of a lifting table whose main requirement is the horizontal lifting and lowering of loads. The corresponding function structure including the FKCs 
resulting from the QFD is shown in extract in Figure 7. According to the requirement, the FKCs shown are limited to the parameters describing the tilting of the table.
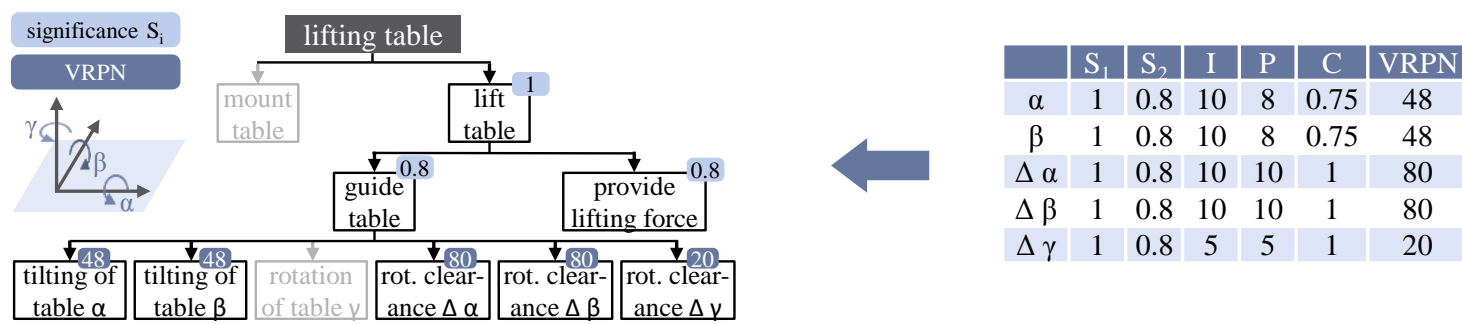

Figure 7. Extract of weighted function structure of lifting table with corresponding VMEA

The parameters for the VMEA required for calculating the weightings are shown in the table on the right in Figure 7. Since the highlighted function and subfunctions are of great importance for the entire product, they are assigned a correspondingly high significance value $S$. The major impact of the rotational clearance and the tilting from the horizontal plane on the superior function "guide table" is represented by the value 10 for $I$. While tilting of the table is likely due to the necessary base $(P=8)$, clearance is unavoidable $(P=10)$ due to the required kinematics. Since $\Delta \gamma$ has a much lower impact on the superior function and rotational clearance around the normal of the table plane is not necessary for the kinematic, the corresponding values are set to 5. Due to the easy detection during the manufacturing process and the possibility for adjustments the factor $C$ for the tilting is contrary to the clearance set to the lowest value 0.75 . The VRPN resulting from the multiplication of these factors is an elementary input in the robustness evaluation matrix for the comparison of different solutions. As an example, the three solutions shown in Figure 8 are taken into account. Although there are numerous other variants, like an alternative drive concept for example, the system is limited to the kinematic for reasons of traceability.

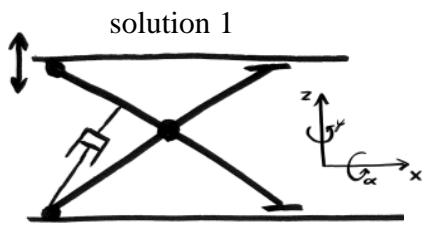

scissor lift table

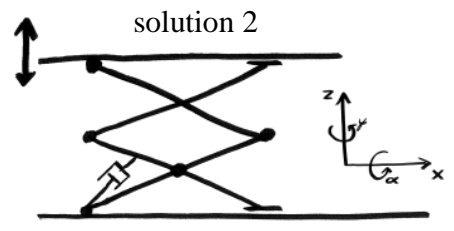

double scissor lift table

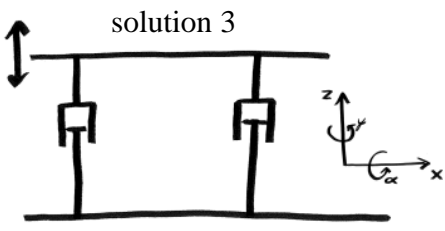

4 hydraulic cylinders

Figure 8. Sketches of the three analyzed solutions

In order to keep the robustness evaluation simple, it is limited to the criteria proposed in Table 1. For the calculation of the RI in the matrix shown in Figure 9, the extent to which the degree of constraint and the number of parameters influence the previously defined FKCs was first evaluated.

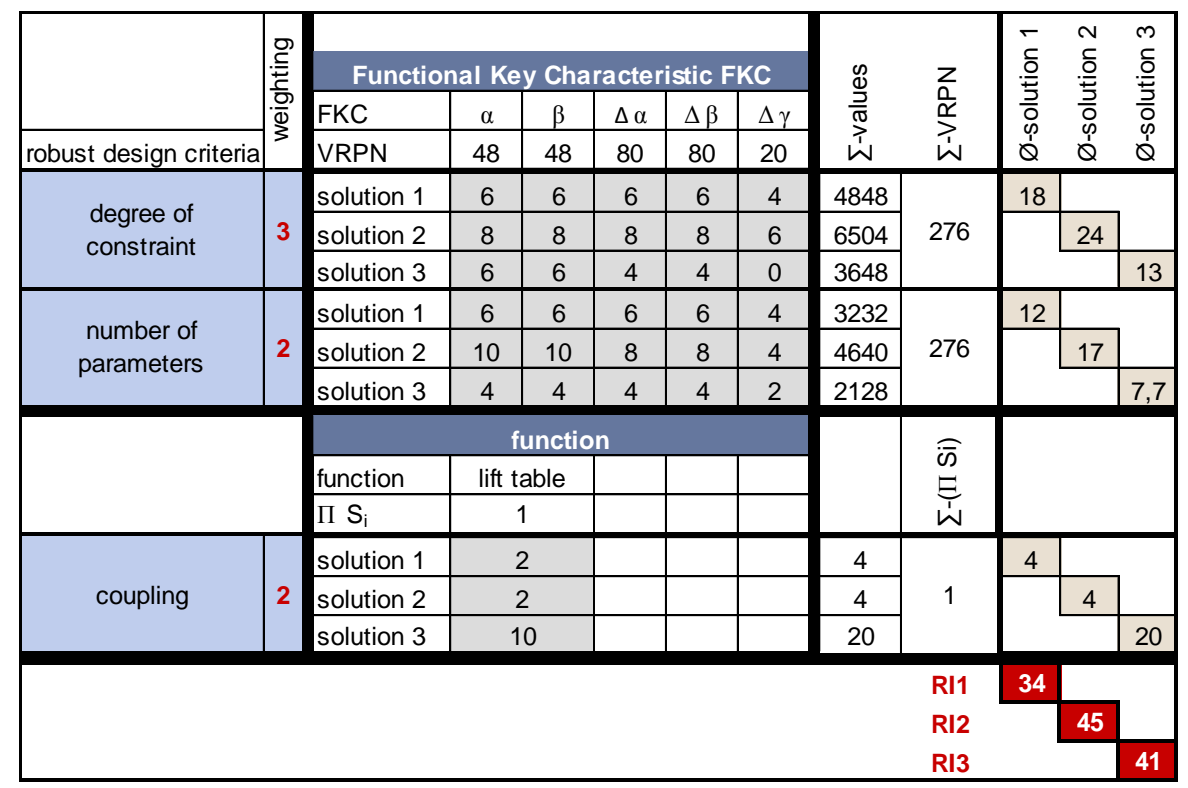

Figure 9. Robustness evaluation matrix for lifting table 
For this purpose, a simplified value table ranging from 0 to 10 was used, with high values indicating low robustness. Since especially the overconstrained system of the complicated solution 2 may lead to clamping of individual parts and therefore has a negative effect on the horizontal position of the table so that it is rated with 10 . The second evaluation is based on the number of parts and contacts contributing to the respective FKCs. Finally, the coupling evaluation at function level considers the separation of the two subfunctions of the function "lift table". Since solution 3 provides both the guidance and the force generation by the four hydraulic cylinders, the strong coupling is to be rated here with 10 . Thus, the resulting RIs indicate a preferred selection of solution 1 , which is in line with the expectations for this simplified case study.

\section{CONCLUSION AND OUTLOOK}

The contribution stresses the lack of quantitative approaches considering tolerances and other fluctuating factors in the early product development stages prior to the geometric definition. Therefore, a novel method and its implementation in a practical tool for supporting the selection of principal solutions in the conceptual design stage with respect to variation and Robust Design aspects is presented. In order to ensure broad applicability, the framework of the method allows individual adaptation depending on the complexity of the product under investigation, the desired quality of the results and the experience of the user. For this purpose, the relevant characteristics (FKCs) are determined on the basis of the requirements as well as the function structure and weighted according to their significance with an adapted VMEA. By breaking it down to the parameter level, the numerous established Robust Design principles can be easily transferred to the concept design and are linked in a common evaluation matrix via weighting. The resulting Robustness Indices enable a smooth integration of the robustness evaluation into the multi-criteria evaluation of concept choice already established in product development process.

Although a thorough documentation of the results means that some steps of the proposed evaluation process no longer have to be completely performed, the procedure is characterized by numerous manual steps. In order to automate these steps and thus further improve the usability of the method, a close link between requirement, function and parameter will be necessary in the future work. In combination with the aforementioned documentation, formalized experience knowledge for the robustness evaluation in the conceptual design stage could be acquired. Furthermore, the approach should be tested in industry in order to identify and overcome current drawbacks with regard to the practical usability.

\section{REFERENCES}

Akao, Y. (2004), Quality function deployment: Integrating customer requirements into product design, Productivity Press, New York.

Almannai, B., Greenough, R. and Kay, J. (2008), “A decision support tool based on QFD and FMEA for the selection of manufacturing automation technologies", Robotics and Computer-Integrated Manufacturing, Vol. 24 No. 4, pp. 501-507. http://doi.org/10.1016/j.rcim.2007.07.002.

Andersson, P. (1997), "On Robust Design in the Conceptual Design Phase. A Qualitative Approach", Journal of Engineering Design, Vol. 8 No. 1, pp. 75-89.

Ayă̆, Z. (2016), "An integrated approach to concept evaluation in a new product development”, Journal of Intelligent Manufacturing, Vol. 27 No. 5, pp. 991-1005. http://doi.org/10.1007/s10845-014-0930-7.

Cheng Lim, P., Tang, N.K.H. and Jackson, P.M. (1999), "An innovative framework for health care performance measurement”, Managing Service Quality: An International Journal, Vol. 9 No. 6, pp. 423-433. http://doi.org/10.1108/09604529910304125.

Dehnad, K. (1989), Quality Control, Robust Design, and the Taguchi Method, Springer US, Boston. http://doi.org/10.1007/978-1-4684-1472-1.

Ebro, M. and Howard, T.J. (2016), "Robust design principles for reducing variation in functional performance", Journal of Engineering Design, Vol. 27 No. 1-3, pp. 75-92. http://doi.org/10.1080/09544828.2015.1103844.

Ebro, M., Howard, T.J. and Rasmussen, J.J. (2012), “The Foundation For Robust Design: Enabling Robustness Through Kinematic Design And Design Clarity”, 12th International Design Conference, Dubrovnik, Marjanovic, D., Storga, M., Pavkovic, N., Bojcetic, N., pp. 817-826.

Engelhardt, R., Birkhofer, H., Kloberdanz, H. and Mathias, J. (2009), "Uncertainty-Mode- and Effects-Analysis - an Approach to Analyze and Estimate Uncertainty in the Product Life Cycle", 17th ICED, Palo Alto, 24.27.08.2009, Norell Bergendahl, M., Grimheden, M., Leifer, L., Skogstad, P., Lindemann, U., pp. 191-202. 
Goetz, S., Schleich, B. and Wartzack, S. (2018), “A new approach to first tolerance evaluations in the conceptual design stage based on tolerance graphs", Procedia CIRP, Vol. 75, pp. 167-172. http://doi.org/10.1016/j.procir.2018.04.030.

Göhler, S.M. (2017), Metric-driven Robust Design - Robustness Quantification of Complex Engineering Systems, PhD Thesis, DTU.

Göhler, S.M. and Howard, T.J. (2015), “The Contradiction Index (CI). A New Metric Combining System Complexity and Robustness for Early Design Stages", 27th International Conference on Design Theory and Methodology, Boston, 02.-05.08.2015, ASME. http://doi.org/10.1115/DETC2015-47255.

Gremyr, I. and Hasenkamp, T. (2011), "Practices of robust design methodology in practice", The TQM Journal, Vol. 23 No. 1, pp. 47-58. http://doi.org/10.1108/17542731111097489.

Haskins, C. (2006), Systems Engineering Handbook, INCOSE.

Heling, B., Schleich, B. and Wartzack, S. (2018), "Robust-Design-Optimization of mechanisms based on kinematic requirements considering uncertainties", Procedia CIRP, Vol. 75, pp. 27-32. http://doi.org/10.1016/j.procir.2018.04.048.

Hermans, J. and Liu, Y. (2013), "Quality Management in the New Product Development. A PPAP Approach”, Quality Innovation Prosperity, Vol. 17 No. 2, http://doi.org/10.12776/QIP.V17I2.150.

Hoffman, H.F. (2014), The Engineering Capstone Course, Springer. http://doi.org/10.1007/978-3-319-05897-9.

Johansson, P., Chakhunashvili, A., Barone, S. and Bergman, B. (2006), "Variation Mode and Effect Analysis. A Practical Tool for Quality Improvement", Quality and Reliability Engineering International, Vol. 22 No. 8 , pp. 865-876. http://doi.org/10.1002/qre.773.

Justel, D., Vidal, R., Arriaga, E., Franco, V. and Val-Jauregi, E. (2007), "Evaluation Method For Selecting Innovative Product Concepts With Greater Potential Marketing Success", 16th ICED, Paris, 28.31.07.2007, Bocquet, J.-C.

Kim-Soon, N. (2012), Quality Management and Practices, InTech. http://doi.org/10.5772/36671.

Krehmer, H., Eckstein, R., Lauer, W., Roelofsen, J., Stöber, C., Troll, A., Zanpf, J., Weber, N., Meerkamm, H., Henrich, A., Lindemann, U., Rieg, F. and Wartzack, S. (2010), "Das Forflow-Prozessmodell zur Unterstützung der multidisziplinären Produktentwicklung”, Konstruktion, Vol. 10, pp. 59-68.

Mathias, J., Kloberdanz, H., Eifler, T., Engelhardt, R., Wiebel, M., Birkhofer, H. and Bohn, A. (2011), "Selection Of Physical Effects Based On Disturbances And Robustness Ratios In The Early Phases Of Robust Design”, 18th ICED, Copenhagen, 15.-19.08.2011, Culley, S.J., Hicks, B.J., McAloone, T.C., Howard, T.J. and Malmqvist, J., pp. 324-335.

Matthiassen, B. (1997), Design for robustness and reliability, $\mathrm{PhD}$ thesis, DTU.

Mc Dermott, R.E., Mikulak, R.J. and Beauregard, M.R. (2009), The basics of FMEA, Productivity Press, New York.

P. Sullivan, L. (1986), “Quality Function Deployment”, Quality Progress, Vol. 19 No. 6, pp. 39-50.

Pahl, G., Beitz, W., Blessing, L., Feldhusen, J., Grote, K.-H. and Wallace, K. (2007), Engineering Design: A Systematic Approach, Springer, London.

Pfeifer, T. (2002), Quality management: Strategies, methods, techniques, Hanser, München.

Schleich, B., Anwer, N., Zhu, Z., Qiao, L., Mathieu, L. and Wartzack, S. (2014), “A Comparative Study on Tolerance Analysis Approaches”, ISoRD14, Kopenhagen, 14.-15.08.2014, pp. 29-39. http://doi.org/10.4122/dtu:2084.

Song, Y. and Su, Q. (2015), "The relationship between quality management and new product development. Evidence from China”, Operations Management Research, Vol. 8 No. 1-2, pp. 1-14. http://doi.org/10.1007/s12063-014-0096-7.

Suh, N.P. (1998), “Axiomatic Design Theory for Systems”, Research in Engineering Design, Vol. 10 No. 4, pp. $189-209$.

Söderberg, R. and Lindkvist, L. (1999), “Computer Aided Assembly Robustness Evaluation”, Journal of Engineering Design, Vol. 10 No. 2, pp. 165-181.

Taguchi, G., Elsayed, E.A. and Hsiang, T.C. (1990), Quality engineering in production systems, McGraw-Hill series in industrial engineering and management science, McGraw-Hill, New York.

Taguchi, G., Yano, H., Chowdhury, S. and Taguchi, S. (Eds.) (2005), Taguchi's quality engineering handbook, John Wiley \& Sons, Hoboken, N.J, Livonia, Mich.

Thornton, A.C. (2004), Variation risk management: Focusing quality improvements in product development and production, Wiley, Hoboken, NJ.

Zare Mehrjerdi, Y. (2010), "Quality function deployment and its extensions”, International Journal of Quality \& Reliability Management, Vol. 27 No. 6, pp. 616-640. http://doi.org/10.1108/02656711011054524.

Zhai, L.-Y., Khoo, L.-P. and Zhong, Z.-W. (2009), "Design concept evaluation in product development using rough sets and grey relation analysis", Expert Systems with Applications, Vol. 36 No. 3, pp. 7072-7079. http://doi.org/10.1016/j.eswa.2008.08.068.

\section{ACKNOWLEDGEMENTS}

The authors thank the German Research Foundation (DFG) for supporting the research project WA 2913/17-1. 